\title{
Morfea: revisión práctica de su diagnóstico, clasificación y
} tratamiento

\author{
Pamela Rodríguez-Salgado y María Teresa García-Romero* \\ Instituto Nacional de Pediatría, Servicio de Dermatología, Ciudad de México, México
}

\section{Resumen}

La morfea o esclerodermia localizada es una enfermedad poco común del tejido conectivo que se manifiesta con esclerosis localizada de la piel y, en algunos casos, con lesiones extracutáneas. Su etiología no se comprende por completo, pero se cree que hay predisposición genética, además de factores ambientales desencadenantes. La clasificación de la enfermedad no es sencilla debido a las múltiples presentaciones, sin embargo, es útil para definir el tratamiento, el cual debe individualizarse e iniciarse tempranamente para evitar complicaciones cosméticas y funcionales. En esta revisión resumimos los aspectos prácticos más importantes de la clasificación, métodos diagnósticos y de evaluación de actividad en morfea, así como las opciones terapéuticas disponibles, con énfasis en la evidencia clínica existente respecto a su eficacia y seguridad.

PALABRAS CLAVE: Morfea. Esclerodermia localizada. Liquen escleroso. Liquen atrófico.

\begin{abstract}
Morphea, or localized scleroderma, is a rare disease of the connective tissue that manifests itself with localized sclerosis of the skin and, in some cases, with extracutaneous manifestations. Its etiology is not fully understood, but it is believed that there is genetic predisposition, in addition to environmental triggering factors. Classification of the disease is not simple due to its multiple presentations; however, it is useful in order to define the treatment, which should be individualized and started early to avoid cosmetic and functional complications. In this review, we summarize the most important practical aspects of the classification, diagnostic methods and evaluation of morphea activity, as well as available therapeutic options, with an emphasis on existing clinical evidence regarding their efficacy and safety.
\end{abstract}

KEY WORDS: Morphea. Localized scleroderma. Lichen sclerosus et atrophicus.

Correspondencia:

*María Teresa García-Romero

E-mail: teregarro@gmail.com
Fecha de recepción: 07-03-2018

Fecha de aceptación: 19-11-2018

DOI: 10.24875/GMM.18004288
Gac Med Mex. 2019;155:522-531

Disponible en PubMed www.gacetamedicademexico.com 


\section{Introducción}

La morfea, también llamada esclerodermia localizada, es una enfermedad inflamatoria crónica de la dermis y el tejido celular subcutáneo en la que se genera un proceso de esclerosis con engrosamiento difuso e induración de la piel, así como atrofia a diversos niveles. ${ }^{1-3}$ Frecuentemente es de evolución benigna, sin embargo, $10 \%$ de los pacientes sufre esclerosis y atrofia severa que pueden provocar deformidad, contracturas o alteraciones en el crecimiento. ${ }^{2,3}$

La morfea se considera una entidad distinta a la esclerosis sistémica que no evoluciona a esta, aunque comparten factores fisiopatogénicos. Frecuentemente los pacientes con morfea pueden tener afección extracutánea, principalmente involucramiento ocular, articular y del sistema nervioso central. ${ }^{2}$

El diagnóstico y manejo de los pacientes con morfea no es siempre fácil y puede ser un reto para el dermatólogo. En este artículo se revisan las generalidades de la enfermedad, con enfoque especial en los aspectos de diagnóstico, clasificación y tratamiento.

\section{Generalidades}

La morfea es relativamente infrecuente, con una incidencia anual de 2.7 por cada 100000 habitantes. ${ }^{4}$ Predomina en mujeres, con una relación de 2.6:15,6 y aunque afecta a todas las razas, predomina en la población caucásica. En 75 a $90 \%$ de los pacientes se presenta entre los 20 y 50 años. ${ }^{1}$ Es posible que debido al retraso en el diagnóstico, la verdadera prevalencia de la morfea se encuentre subestimada. ${ }^{3}$

La etiología y patogénesis de la morfea aún no se comprenden completamente. Se piensa que están involucrados factores autoinmunes, ambientales y diversos eventos que desencadenan la producción de citoquinas; como pueden ser infecciones, radiación o trauma. ${ }^{3,7}$ La infección por Borrelia burgdorferi podría tener un papel patogénico, sin embargo, no se ha confirmado la asociación causal. ${ }^{7}$ Existe la hipótesis no probada de que la morfea pueda deberse a un mosaicismo poscigótico por la distribución blascoide o dermatómica de muchas lesiones ${ }^{8}$ (Figura 1).

El proceso de esclerosis de la piel comprende tres eventos principales, los cuales pueden ser blancos terapéuticos:

- Una lesión primaria microvascular, con elevación de SVCAM-1 y sE-selectin, indicadores de activación endotelial, además de expresión de

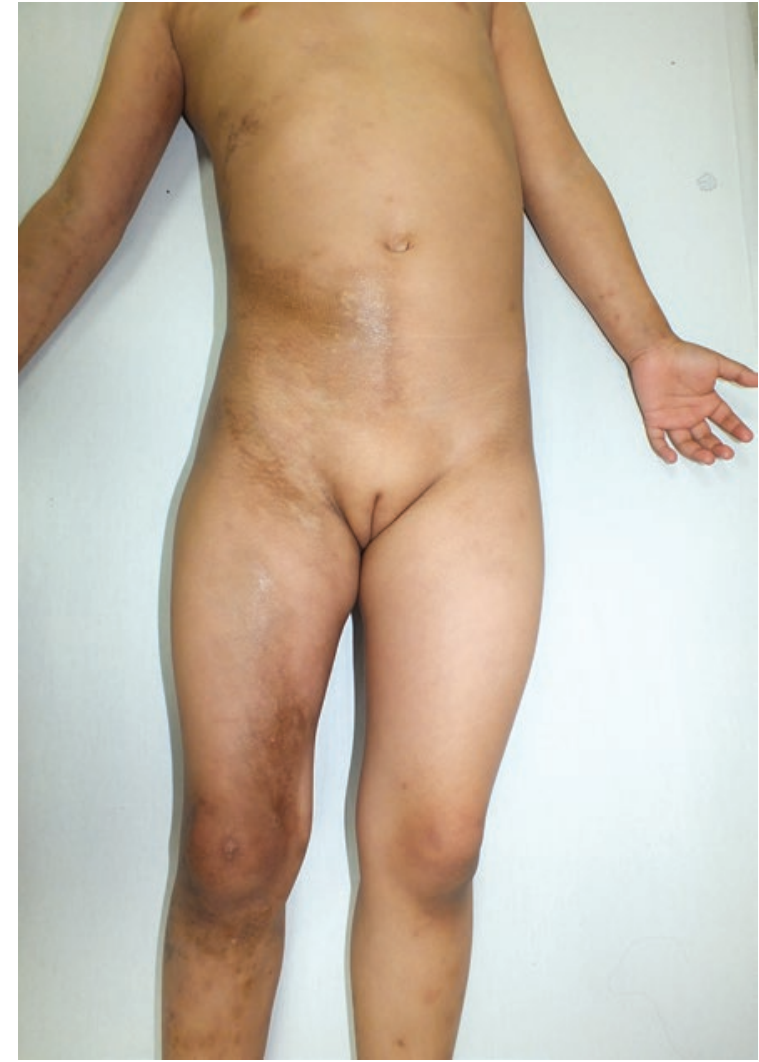

Figura 1. Paciente con morfea generalizada con múltiples placas induradas e hiperpigmentadas con distribución blascoide.

moléculas de adhesión, engrosamiento de la membrana basal e hiperplasia de la íntima. ${ }^{9}$

- Control de la función de fibroblastos por células T CD4+ perivasculares que producen interleucina-4 y factor de crecimiento transformador $\beta$; citocinas que dirigen la diferenciación hacia el fenotipo Th2, reclutan eosinófilos (los cuales se pueden encontrar en las fases tempranas de morfea) y modifican la síntesis de colágeno por fibroblastos. ${ }^{10}$

- Producción patológica de colágeno (I, II, III) por los fibroblastos, así como otras proteínas de matriz extracelular. ${ }^{3}$

\section{Clasificación y cuadro clínico}

La morfea incluye una variedad de condiciones en las que la piel o el tejido celular subcutáneo están afectados por esclerosis. La clasificación puede orientar al tratamiento y pronóstico, sin embargo, no es sencilla debido a que los diferentes tipos no son siempre claros y se sobreponen; ${ }^{11}$ la más aceptada actualmente se detalla en la Tabla $1 .{ }^{12}$

La morfea puede afectar cualquier parte del cuerpo y se caracteriza por una o varias placas de piel 
Tabla 1. Clasificación actual de la morfea según la Sociedad Europea de Reumatología Pediátrica, 20049

\begin{tabular}{|c|c|c|}
\hline Subtipo & Modalidad & Clínica \\
\hline \multirow[t]{2}{*}{ Morfea circunscrita o en placas } & Superficial & $\begin{array}{l}\text { Lesiones ovales-redondas únicas o múltiples limitadas a la epidermis y } \\
\text { dermis. }\end{array}$ \\
\hline & Profunda & $\begin{array}{l}\text { Lesiones ovales-redondas únicas o múltiples que involucran tejido celular } \\
\text { subcutáneo, fascia o músculo. }\end{array}$ \\
\hline \multirow[t]{2}{*}{ Morfea lineal } & Tronco/extremidades & Lesiones lineales que involucran dermis, tejido subcutáneo o profundo. \\
\hline & Cabeza & $\begin{array}{l}\text { En golpe de sable, atrofia hemifacial progresiva, lesiones lineales de la } \\
\text { cara (puede involucrar al hueso subyacente). }\end{array}$ \\
\hline Morfea generalizada & & 4 o más placas, en 2 o más sitios anatómicos. \\
\hline $\begin{array}{l}\text { Morfea panesclerótica inhabilitante } \\
\text { de la infancia }\end{array}$ & & $\begin{array}{l}\text { Lesión circunferencial de la mayoría del área de superficie } \\
\text { corporal (respetando la punta de los dedos y ortejos) que afecta la piel, } \\
\text { tejido celular subcutáneo, músculo o hueso. Sin involucramiento visceral. }\end{array}$ \\
\hline Morfea mixta & & Combinación de cualquiera de los subtipos. \\
\hline
\end{tabular}

endurecida, brillante, atrófica, de tamaño variable, bien delimitadas, hiper o hipopigmentadas. Las placas comienzan insidiosamente como áreas eritemato-edematosas o violáceas y se expanden de manera centrífuga; generalmente son asintomáticas y pasan desapercibidas por el paciente. Conforme evolucionan, comienzan a transformarse en tejido esclerótico, indurado, adquieren un color blanco brillante y en la periferia, anillos violáceos que desaparecen al detenerse la progresión de la lesión y dan lugar a cambios pigmentarios residuales (Figura 2). Evolucionan en forma crónica hasta que la atrofia alcanza diversos niveles: epidermis, dermis o tejido celular subcutáneo. En ocasiones se combinan distintas formas clínicas en un mismo paciente. La actividad de las lesiones dura de tres a cinco años, con tendencia a la remisión, sin embargo, puede haber recurrencias. En los niños se presentan formas más graves, mutilantes y discapacitantes, como la morfea panesclerótica inhabilitante de la infancia. ${ }^{3,13,14}$

La morfea lineal es la variante más frecuente durante la infancia o adolescencia, inicia en esta etapa en 40 a $70 \%$ de los pacientes. ${ }^{11}$ Afecta principalmente extremidades, cabeza en área frontal (morfea en golpe de sable) (Figura 2) y tórax. Se acompaña de pérdida de anexos, prurito y xerosis. Cerca de $50 \%$ de los pacientes con este tipo de morfea presenta también morfea en placas (morfea mixta), frecuente en niños y rara en adultos. ${ }^{5,6,15}$ Hasta $30 \%$ de los pacientes con morfea lineal pueden presentar afección extracutánea con alteraciones oftalmológicas o neurológicas como migrañas, cefaleas, convulsiones
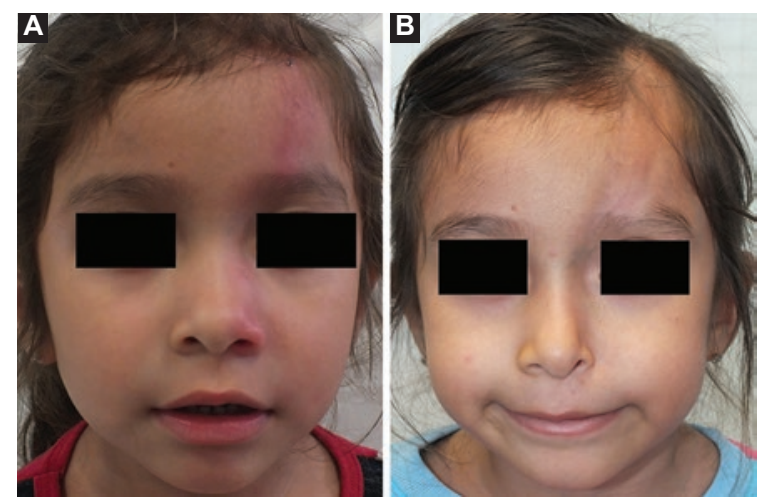

Figura 2. A) Placa eritemato-edematosa en línea paramedial de cara que corresponde a una lesión inicial de morfea lineal. B) La misma lesión varios meses después, donde es evidente la esclerosis, atrofia y pérdida de anexos.

o uveítis; o musculoesqueléticas en pacientes con afección en extremidades. ${ }^{15,16}$

La morfea circunscrita o en placas es el tipo más frecuente en los adultos y la forma más superficial debido a la limitación del proceso fibrótico a la dermis. Se caracteriza por placas escleróticas circunscritas u ovales, únicas o múltiples, de 1 a $30 \mathrm{~cm}$ (Figura 3). 3,13

La morfea generalizada es una forma severa de la enfermedad, definida como cuatro o más placas de más de $3 \mathrm{~cm}$ que confluyen e involucran más de dos sitios anatómicos (Figura 1). Se presenta en 7 a $9 \%$ de los pacientes con morfea y se caracteriza por extensión rápida de las placas. ${ }^{13}$ Por lo general no se asocia con manifestaciones extracutáneas. ${ }^{11}$

La morfea panesclerótica (también llamada inhabilitante de la infancia) es una variante agresiva y mutilante que con mayor frecuencia afecta a niños. La presentación es rápidamente progresiva y deteriora 


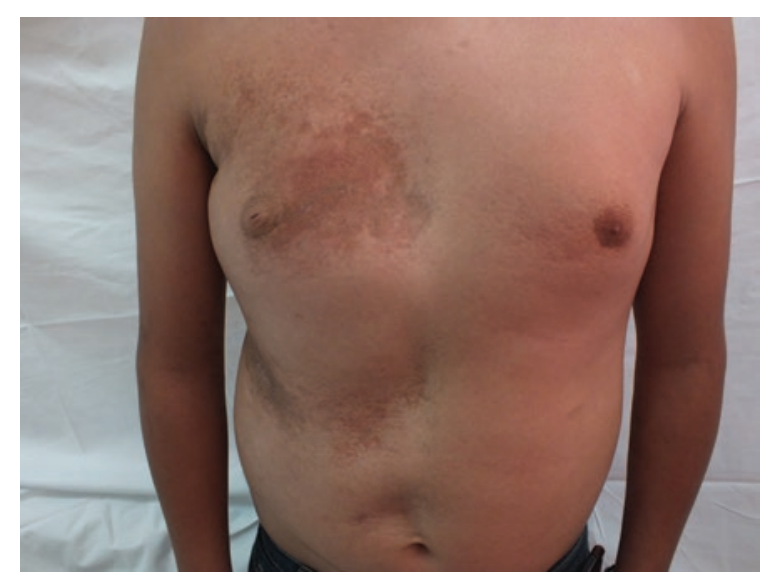

Figura 3. Morfea en placas en tronco. Es notoria la atrofia superficial epidérmica y dérmica con vasos visibles.

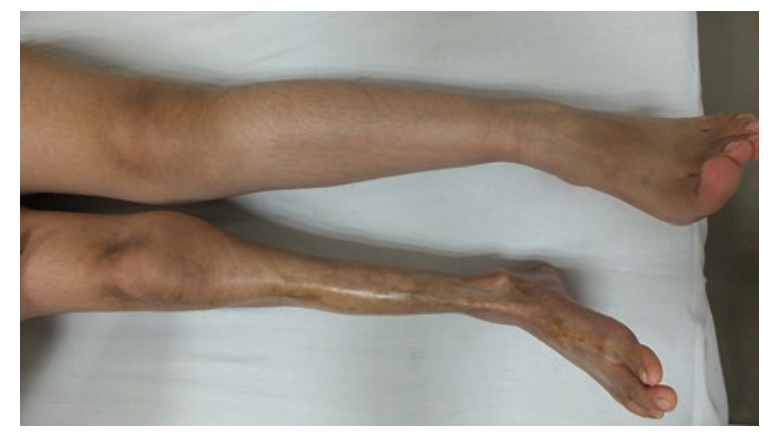

Figura 4. Morfea panesclerótica de la infancia en extremidad inferior izquierda. Nótese la afección circunferencial y profunda de la esclerosis y la atrofia.

estructuras profundas como músculos, tendones y hueso, al generar contracturas articulares, ulceraciones dolorosas y calcificaciones que llevan a incapacidad importante (Figura 4). ${ }^{14,17}$

Algunas variantes menos comunes de morfea se describen en la Tabla 2.3,5,14,16,18,19

\section{Diagnóstico}

El diagnóstico de morfea es principalmente clínico, mediante historia clínica y examen físico cuidadoso de la piel. El interrogatorio al paciente sobre la evolución de las lesiones o la comparación con fotografías previas es de mucha ayuda, así como identificar los diferentes patrones de morfología: en placa o circunscrita, lineal, generalizada, superficial o profunda. ${ }^{19-22}$

\section{Estudios de laboratorio}

Los exámenes de laboratorio no se consideran necesarios para el diagnóstico o el seguimiento de los pacientes con morfea. La velocidad de sedimentación globular y la biometría hemática se encuentran en
Tabla 2. Variantes menos comunes de la morfea $a^{1,11,19}$

\begin{tabular}{ll} 
Morfea guttata & $\begin{array}{l}\text { Lesiones escleroatróficas blanquecinas y } \\
\text { pequeñas que carecen del carácter firme de } \\
\text { la morfea, con orificios foliculares dilatados. }\end{array}$ \\
$\begin{array}{ll}\text { Morfea } \\
\text { nodular (queloide) }\end{array}$ & $\begin{array}{l}\text { Lesiones nodulares que se asemejan a } \\
\text { queloides. }\end{array}$ \\
\hline Atrofoderma & $\begin{array}{l}\text { Múltiples placas sutilmente deprimidas, } \\
\text { ovales e hiperpigmentadas aparecen en el } \\
\text { tronco y miembros superiores, sin presencia } \\
\text { de esclerosis. }\end{array}$ \\
\hline $\begin{array}{l}\text { Síndrome de } \\
\text { Parry-Romberg o } \\
\text { hemiatrofia facial } \\
\text { progresiva }\end{array}$ & $\begin{array}{l}\text { Atrofia uniforme de la dermis, tejido celular } \\
\text { subcutáneo y muscular y, en algunos casos, } \\
\text { hueso, en una distribución hemifacial o }\end{array}$ \\
$\begin{array}{ll}\text { trigeminal. Se asocia con sintomatología } \\
\text { neurológica hasta en } 30 \text { \% de los casos. }\end{array}$ \\
$\begin{array}{l}\text { Fascitis } \\
\text { eosinofílica o } \\
\text { síndrome de } \\
\text { Shulman }\end{array}$ & $\begin{array}{l}\text { De inicio súbito, con induración dolorosa } \\
\text { bilateral exclusivamente de las extremidades. } \\
\text { Se acompaña de eosinofilia periférica y } \\
\text { aumento de la sedimentación eritrocítica. Es } \\
\text { poco frecuente en la edad infantil. }\end{array}$ \\
\hline $\begin{array}{l}\text { Liquen escleroso y y } \\
\text { atrófico }\end{array}$ & $\begin{array}{l}\text { Placas atróficas, color blanco o lila pálido } \\
\text { de aspecto brillante. Afecta frecuentemente } \\
\text { el área anogenital, pero se puede presentar } \\
\text { en cualquier área del cuerpo. Asociada con } \\
\text { morfea circunscrita y generalizada. }\end{array}$ \\
\hline
\end{tabular}

rangos normales en la mayoría de los casos, pero puede aparecer eosinofilia, especialmente en etapas tempranas de la enfermedad. La presencia de anticuerpos antinucleares o anticuerpos antiADN de doble cadena y antihistona ocurre en aproximadamente en $30 \%$ de los pacientes, principalmente en aquellos con morfea lineal o generalizada. $5,6,13,15,23$

Debido a la frecuencia de alteraciones extracutáneas, especialmente en pacientes con morfea lineal, es importante buscarlas intencionadamente mediante interrogatorio a los pacientes e interconsulta a oftalmología, neurología u ortopedia, además de exámenes paraclínicos pertinentes. Algunos autores recomiendan realizar imagen por resonancia magnética nuclear cerebral y exploración oftalmológica de rutina a pacientes con morfea lineal en cabeza. ${ }^{16}$

\section{Histopatología}

La histología de la morfea depende de dos factores: la etapa de la enfermedad y la profundidad a la que se extiende. En etapas iniciales se encuentra infiltrado inflamatorio linfocitario perivascular, edema endotelial $y$, en ocasiones, eosinófilos, células plasmáticas y mastocitos, así como atrofia de anexos y vasos con paredes engrosadas con luz estrecha (Figura 5). ${ }^{24}$ En etapas tardías, el infiltrado inflamatorio disminuye o 


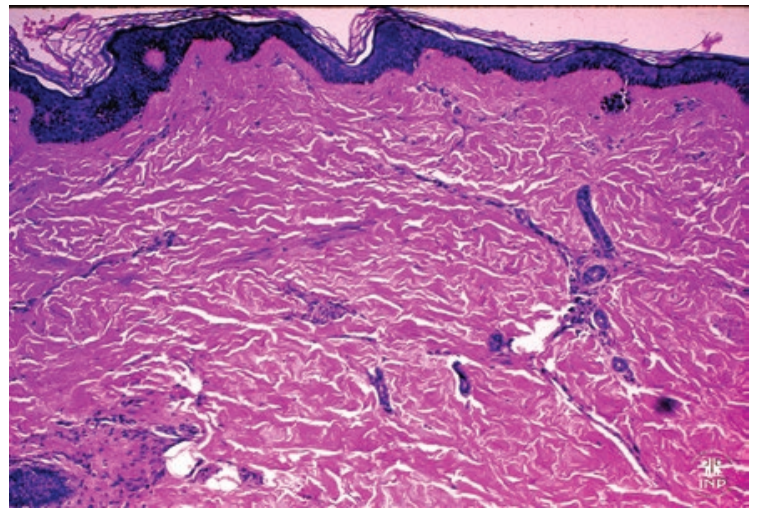

Figura 5. Imagen histológica de morfea en la que se observa hialinización del colágeno dérmico, pérdida de anexos e infiltrado inflamatorio mixto.

desaparece, se observa pérdida de anexos, unión dermoepidérmica aplanada y haces de colágeno en la dermis reticular, con aspecto empaquetado y alineados paralelamente a la unión dermoepidérmica. ${ }^{24} \mathrm{La}$ morfea profunda afecta primariamente al tejido celular subcutáneo, produce esclerosis extensa y hialinización, que se extiende hacia la fascia subyacente. ${ }^{25}$

\section{Evaluación de la actividad y severidad de la morfea}

Evaluar la actividad de la enfermedad es muy importante para tomar decisiones terapéuticas, sin embargo, no siempre es fácil ya que actualmente no existen medidas de resultado objetivas, claras 0 estandarizadas. ${ }^{26}$ Los factores y características referidos en la Tabla 3 pueden ser de ayuda para determinar la actividad de una placa de morfea., ${ }^{1,3,27}$

Se han desarrollado algunas escalas para evaluar la actividad de la enfermedad, como el Índice de Severidad (LoSSI, Localized Scleroderma Severity Index) y el Índice de Daño en Esclerodermia Localizada (LoSDI, Localized Scleroderma Damage Index); juntos conforman la Herramienta de Evaluación Cutánea en Esclerodermia Localizada (LoSCAT, Localized Scleroderma Cutaneous Assessment Tool), la cual ha sido validada (Tabla 4). ${ }^{28,29}$ La escala DIET es otra propuesta para valorar una placa de morfea, sin embargo, no ha sido validada (Tabla 5). ${ }^{27}$

Existe correlación significativa entre las diferentes escalas de actividad de la morfea (LoSSI, LoSCAT, DIET) e incluso con una escala visual análoga o subjetiva de actividad de las lesiones por parte del médico y del paciente, ${ }^{27}$ por ello es aceptable que el médico utilice la que encuentre más práctica para evaluar a sus pacientes.
Tabla 3. Características y factores para evaluar actividad y severidad de la morfea ${ }^{27,30}$

Aparición de nuevas lesiones o expansión de lesiones existentes en los últimos 3 meses.

Eritema o edema moderado o severo en las lesiones.

Bordes eritematovioláceos en las lesiones.

Incremento progresivo de la induración o esclerosis en la lesión.

Pérdida de pelo asociada con las lesiones.

Documentación por el médico de actividad de enfermedad o progresión a tejidos profundos, mediante escalas de actividad, fotografías, resonancia magnética nuclear o ultrasonido.

Biopsia de piel que demuestra actividad de la enfermedad.

Otras herramientas propuestas para evaluar la actividad de la morfea son el plicómetro o durómetro (que valoran el espesor de la piel), una escala computarizada que calcula el área exacta de induración, el ultrasonido Doppler y la termografía infrarroja. ${ }^{30}$

\section{Tratamiento}

El tratamiento de la morfea puede ser un reto, ya que no existe un medicamento que se considere el estándar de oro. Además, la elección del tratamiento dependerá de qué tan activa está la enfermedad, su extensión, localización, profundidad y progresión; es decir, será individualizado a cada paciente (Figura 1). ${ }^{26}$ Debe iniciarse tempranamente, antes de la aparición de complicaciones en pacientes con riesgo de morbilidad asociada por las características de la morfea. ${ }^{31}$ Se recomienda continuarlo hasta que desaparezca la actividad de la enfermedad, aunque la atrofia y despigmentación no mejoren por completo. 111,19,22,32-36

\section{Tratamiento tópico}

El tratamiento tópico está limitado a las variedades superficiales y circunscritas de morfea, sin ninguna restricción en el movimiento o crecimiento. ${ }^{26,37}$ Las opciones terapéuticas, el nivel de evidencia y la fuerza de recomendación se enlistan en la Tabla 6:

- Tacrolimus (0.1\%): inhibidor de calcineurina, con buena efectividad y seguridad. 38,39

- Imiquimod: inmunomodulador que regula al interferón (IFN) $\alpha$ y $\gamma$ e inhibe la producción de colágeno por los fibroblastos mediante la regulación negativa del factor de crecimiento transformador $\beta$. Es particularmente efectivo en lesiones induradas. ${ }^{40}$ 
Tabla 4. LoSCAT, Localized Scleroderma Cutaneous Assessment Tool (Herramienta para la Evaluación Cutánea en Esclerodermia Localizada)

\begin{tabular}{|c|c|c|c|c|c|}
\hline \multicolumn{3}{|c|}{$\begin{array}{l}\text { mLosSI } \\
\text { (Modified Localized Scleroderma Skin Activity Index) }\end{array}$} & \multicolumn{3}{|c|}{$\begin{array}{l}\text { LoSDI } \\
\text { (Localized Scleroderma Skin Damage Index) }\end{array}$} \\
\hline $\begin{array}{l}\text { Nueva lesión o crecimiento } \\
\text { (en último mes) } \\
0=\text { No } \\
3 \text { = Sí }\end{array}$ & $\begin{array}{l}\text { Eritema } \\
0=\text { No } \\
1=\text { Rosado } \\
2=\text { Rojo } \\
3=\text { Rojo Oscuro/violáceo }\end{array}$ & $\begin{array}{l}\text { Engrosamiento } \\
0=\text { No } \\
1=\text { Leve } \\
2=\text { Moderado } \\
3=\text { Severo }\end{array}$ & $\begin{array}{l}\text { Atrofia dérmica } \\
0=\text { No } \\
1=\text { Superficie brillosa } \\
2=\text { Vasos visibles } \\
3=\text { Escalonamiento }\end{array}$ & $\begin{array}{l}\text { Atrofia subcutánea } \\
0=\text { No } \\
1 \text { = Plano } \\
2 \text { = Cóncavo } \\
3 \text { = Atrofia severa }\end{array}$ & $\begin{array}{l}\text { Dispigmentación } \\
0=\text { No } \\
1=\text { Leve } \\
2=\text { Moderada } \\
3=\text { Severa }\end{array}$ \\
\hline $0-3$ & $0-3$ & $0-3$ & $0-3$ & $0-3$ & $0-3$ \\
\hline
\end{tabular}

Tabla 5. Escala DIET (dispigmentación, induración, eritema y telangiectasis)

\begin{tabular}{|c|c|}
\hline Dispigmentación (hipo o hiper) & $0-3$ \\
\hline Induración & $0-3$ \\
\hline Eritema & $0-3$ \\
\hline Telangiectasias & $0-3$ \\
\hline
\end{tabular}

Tabla 6. Tratamientos tópicos para morfea

\begin{tabular}{|c|c|c|}
\hline Tratamiento & $\begin{array}{l}\text { Nivel de } \\
\text { evidencia* }\end{array}$ & $\begin{array}{l}\text { Grado de } \\
\text { recomendación* }\end{array}$ \\
\hline Imiquimod $5 \%{ }^{40}$ & $1 b$ & B \\
\hline Tacrolimus ${ }^{39}$ & $1 b$ & B \\
\hline Pirfenidona ${ }^{43}$ & 4 & C \\
\hline Calcipotrio| $^{42}$ & 4 & C \\
\hline $\begin{array}{l}\text { Calcipotriol/dipropionato de } \\
\text { betametasona }{ }^{41}\end{array}$ & 4 & C \\
\hline Calcipotriol/PUVA crema70 & 5 & D \\
\hline
\end{tabular}

- Calcipotriol: derivado de la vitamina D, in vitro inhibe la proliferación de fibroblastos; clínicamente es efectivo solo y combinado con dipropionato de betametasona. ${ }^{41,42}$

Otros medicamentos utilizados anecdóticamente y en pequeñas series de pacientes con aparente beneficio son la pirfenidona y la inmunoglobulina subcutánea. ${ }^{25,43}$ Se ha demostrado con buena evidencia (nivel $1 b$ ) que el IFN $\gamma$ intralesional no es efectivo. ${ }^{44}$

Los esteroides tópicos, utilizados por más de $90 \%$ de médicos, no han sido estudiados formalmente. ${ }^{36}$

\section{Tratamiento sistémico}

Debe utilizarse en morfea lineal en cabeza y extremidades con potencial incapacidad funcional o cosmética, en lesiones generalizadas o rápidamente progresivas y en morfea panesclerótica. ${ }^{1,3,11,19,21,22,26,32-35,37,45,46}$ Las opciones terapéuticas, el nivel de evidencia y la fuerza de recomendación se enlistan en la Tabla 7; las más eficaces son las siguientes:

- Metotrexate: inhibidor de la tetrahidrofolato reductasa. Es el medicamento con mayor evidencia en cuanto a su efectividad en morfea y actualmente se considera el tratamiento de primera línea, con o sin corticosteroides sistémicos por un corto periodo de tiempo (prednisona 0.5$1 \mathrm{mg} / \mathrm{kg} / \mathrm{día}$ ) o en pulsos (metilprednisolona intravenosa 1-2 mg/kg/día por tres días de cada mes por tres meses). El metotrexate debe continuarse por lo menos 12 a 18 meses y suspenderse hasta que hayan pasado seis meses sin actividad de la enfermedad. ${ }^{47-51}$

- Fototerapia: con luz ultravioleta (UV) A1, UV B o con psoraleno (PUVA) se altera el curso de la enfermedad, con resolución o mejoría notoria en los pacientes. Induce la expresión de metaloproteinasa 1 , una colagenasa que reduce el procolágeno y colágeno en la piel y es efectiva en todos los subtipos de morfea. Se ha encontrado que UVA1 a distintas dosis (baja, media y alta) y UVA banda ancha son eficaces, si bien es menos claro el beneficio respecto al riesgo del PUVA vía oral, baño o tópico. ${ }^{52-59}$ La UVB de banda angosta ha demostrado también ser efectiva y con un perfil de seguridad muy bueno..$^{57}$

Existe evidencia de que la prednisona como monoterapia (nivel $2 b)^{47}$ y el calcitriol 1,25 dihidroxivitamina D3 vía oral (nivel $1 b)^{60,61}$ no son tratamientos efectivos.

\section{Terapia física}

La fisioterapia es obligatoria en los pacientes en quienes la morfea genera alteraciones en la movilidad o incapacidad y se considerada parte del tratamiento 
Tabla 7. Tratamientos sistémicos para morfea

\begin{tabular}{|c|c|c|}
\hline Tratamiento & $\begin{array}{c}\text { Nivel de } \\
\text { evidencia* }\end{array}$ & $\begin{array}{l}\text { Grado de } \\
\text { recomendación* }\end{array}$ \\
\hline Metotrexate ${ }^{47}$ & $1 b$ & A \\
\hline UVA1 DB ${ }^{57}$ & $1 b$ & A \\
\hline UVA1 DM ${ }^{57}$ & $1 b$ & A \\
\hline UVA1 DA ${ }^{57}$ & $2 b$ & B \\
\hline UVA1 DB/calcipotriol & $2 b$ & B \\
\hline NB UVB ${ }^{57}$ & $2 b$ & B \\
\hline $\begin{array}{l}\text { Láser fraccionado } \mathrm{CO}_{2} \text { versus } \\
\text { UVA1 } \mathrm{DB}^{71}\end{array}$ & $2 b$ & B \\
\hline PUVA vía oral72 & 4 & C \\
\hline PUVA baño/tópico ${ }^{58}$ & 4 & C \\
\hline Abatacept ${ }^{73}$ & 4 & C \\
\hline Ciclosporina ${ }^{74}$ & 4 & C \\
\hline Micofenolato de mofetilo $0^{75,76}$ & 4 & C \\
\hline Tocilizumab ${ }^{77}$ & 4 & C \\
\hline PUVA/ácido retinoico & 4 & C \\
\hline D-penicilamina ${ }^{78}$ & 4 & C \\
\hline Imatimib ${ }^{79,80}$ & 5 & $D$ \\
\hline Metotrexate+láser excimer ${ }^{81}$ & 5 & D \\
\hline Leflunomida/infliximab ${ }^{82}$ & 5 & $D$ \\
\hline Etretinato $^{83}$ & 5 & D \\
\hline Fotoféresis EC+UVA BB ${ }^{84}$ & 5 & D \\
\hline \multicolumn{3}{|c|}{$\begin{array}{l}\mathrm{CO}_{2}=\text { dióxido de carbono, UVA1 = fototerapia con luz ultravioleta } \mathrm{A} 1(\mathrm{DB}=\text { dosis baja } \\
\left.\text { menos de } 30 \mathrm{~J} / \mathrm{cm}^{2}, \mathrm{DM}=\text { dosis media } 50-70 \mathrm{~J} / \mathrm{cm}^{2}, \mathrm{DA}=\text { dosis alta } 130 \mathrm{~J} / \mathrm{cm}^{2}\right) \text {, } \\
\mathrm{NB} \text { UVB = fototerapia con luz ultravioleta B Be banda estrecha, } \\
\text { PUVA = psoraleno + fototerapia con luz ultravioleta } \mathrm{A}, \mathrm{EC}=\text { extracorpórea, } \\
\text { UVA BB = fototerapia con luz ultravioleta } \mathrm{A} \text { en banda ancha. } \\
\text { *De acuerdo con el Centro de Medicina Basada en Evidencia, Oxford (anexo 1). }\end{array}$} \\
\hline
\end{tabular}

en pacientes con morfea lineal en extremidades 0 generalizada extensa, para preservar el grado de movilidad y minimizar las contracturas, si bien su efectividad nunca ha sido estudiada. ${ }^{35}$

\section{Tratamiento quirúrgico o cosmético}

Una vez que la enfermedad esté inactiva sin tratamiento por lo menos por uno o dos años y los niños hayan alcanzado el crecimiento total, el daño residual puede ser tratado de diversas maneras como cirugía plástica, lipoimplantes, material de relleno o colgajos libres de tejido graso. ${ }^{62-65} \mathrm{El}$ objetivo del tratamiento quirúrgico es específicamente la restauración de volumen, ya que la asimetría es una de las principales preocupaciones de los pacientes. ${ }^{66}$ La cirugía ortopédica puede estar indicada si los pacientes desarrollan deformidad de las articulaciones y los huesos como secuelas de la morfea lineal o profunda, incluyendo la liberación de contracturas articulares y procedimientos de alargamiento de extremidades. ${ }^{67,68}$

\section{Pronóstico}

El pronóstico de la morfea circunscrita o superficial es bueno, aunque la enfermedad presenta tendencia hacia la progresión. Generalmente la enfermedad se inactiva entre los tres a cinco años, pero aproximadamente $30 \%$ de los pacientes presentará recidivas, ${ }^{38}$ especialmente si la morfea inició en la niñez y si el tratamiento no fue adecuado. Las formas lineales y profundas pueden dejar secuelas importantes con discapacidad funcional y estética.

\section{Conclusiones}

La morfea es una enfermedad inflamatoria de inicio frecuente en la infancia y de predominio en mujeres, en la que se produce esclerosis de la piel y tejidos subyacentes de forma localizada. Su fisiopatología no es del todo comprendida, sin embargo, se conoce que hay daño microvascular inicial y respuesta anormal por linfocitos $T$ que genera producción anómala de colágena y fibrosis. En algunos casos su clasificación resulta difícil, pero siempre debe ponderarse la localización y el grado de incapacidad potencial que puede generar la enfermedad en el paciente, para escoger el tratamiento, el cual debe individualizarse e iniciarse prontamente. El tratamiento tópico se prefiere para las formas circunscritas o localizadas; el tacrolimus y el imiquimod son las opciones más recomendadas. La terapia sistémica es de elección para las formas lineales o severas; el metotrexate y la fototerapia con UVA1 se consideran las opciones de primera línea. Aproximadamente $30 \%$ de los pacientes con morfea lineal puede tener lesiones extracutáneas neurológicas, articulares u oftalmológicas, que deben buscarse intencionadamente y descartarse.

\section{Bibliografía}

1. Careta MF, Romiti R. Localized scleroderma: clinical spectrum and therapeutic update. An Bras Dermatol. 2015;90:62-73

2. García de la Peña-Lefebvre P. Esclerodermia localizada. Protoc Diagn Ter Pediatr. 2014:1:1-6.

3. García-Romero M, Laxer RM, Pope E. Localized scleroderma: symptoms, diagnosis and treatment. En: De Winter R, editor. Scleroderma symptoms, diagnosis and treatment. EE. UU.: Nova Science Publishers; 2013.

4. Peterson L, Nelson A, Su W, et al. The epidemiology of morphea (localized scleroderma) in Olmsted County 1960-1993. J Rheumatol. 1997; 24:73-80. 
5. Zulian F, Athreya BH, Laxer RM, Nelson AM, Feitosa-De Oliveira SK Punaro MG, et al. Juvenile localized scleroderma: clinical and epidemiological features in 750 children. An international study. Rheumatology (Oxford). 2006;45:614-620.

6. Christen-Zaech S, Hakin MD, Afsar FS, Paller AS. Pediatric morphea (localized scleroderma): review of 136 patients. J Am Acad Dermatol. 2008;59:385-396

7. Alfaro-Cordero K. Morfea: el desequilibrio entre la producción y la destrucción del colágeno. Rev Med Cos. 2016;73:351-354.

8. Paller AS. Piecing together the puzzle of cutaneous mosaicism. J Clin Invest. 2004;114:1407-1409.

9. Yamane K, Ihn H, Kubo M, Yazawa N, Kikuchi K, Soma Y, et al. Increased serum levels of soluble vascular cell adhesion molecule 1 and E-selectin in patients with localized scleroderma. J Am Acad Dermatol. 2000;42:64-69.

10. Serpier H, Gillery P, Salmon-Ehr V, Garnotel R, Georges N, Kalis B, et al. Antagonistic effects of interferon-gamma and interleukin-4 on fibroblast cultures. J Invest Dermatol. 1997:109:158-162.

11. Bielsa Marsol I. Update on the classification and treatment of localized scleroderma. Act Dermosifiliogr. 2013;104:654-666.

12. Zulian F, Woo P, Athreya BH, Laxer RM, Medsger TA, Lehman TJ, et al. The Pediatric Rheumatology European Society/American College of Rheumatology/European League Against Rheumatism Provisional Classification Criteria for Juvenile Systemic Sclerosis. Arthritis Rheum. 2007; 57:203-212.

13. Noguera M, Sauro-De Carvalho M, Lombardi V. Morfea. Arch Argent Dermatol. 2012;62:77-84.

14. Zulian F, Cuffaro G, Sperotto F. Scleroderma in children: an update. Curr Opin Rheumatol. 2013;25:643-650.

15. Pequet M, Holland KE, Zhao S, Drolet BA, Galbraith SS, Siegel DH, et al Risk factors for morphoea disease severity: a retrospective review of 114 paediatric patients. Br J Dermatol. 2014;170:895-900.

16. Chiu YE, Vora S, Kwon EK, Maheshwari M. A significant proportion of children with morphea en coup de sabre and Parry-Romberg syndrome have neuroimaging findings. Pediatr Dermatol. 2012;29:738-748.

17. Kim A, Marinkovich N, Vásquez R, Jacobe HT. Clinical features of patients with morphea and the pansclerotic subtype: a cross-sectional study from the morphea in adults and children cohort. J Rheumatol. 2014;41:106-112.

18. Kreuter A, Wischnewski J, Terras S, Altmeyer P, Stücker M, Gambichler T. Coexistence of lichen sclerosus and morphea: a retrospective analysis of 472 patients with localized scleroderma from a German tertiary referral center. J Am Acad Dermatol. 2012;67:1157-1162.

19. Pope E, Laxer RM. Diagnosis and management of morphea and lichen sclerosus and atrophicus in children. Pediatr Clin North Am. 2014;61:309-319.

20. Fett NM. Morphea (localized scleroderma). JAMA Dermatol. 2013; 149:1124.

21. Knobler R, Moinzadeh $P$, Hunzelmann N, Kreuter A, Cozzio A, Mouthon L, et al. European Dermatology Forum S1-Guideline on the diagnosis and treatment of sclerosing diseases of the skin, part 1: localized scleroderma, systemic sclerosis and overlap syndromes. J Eur Acad Dermatol Venereol. 2017;31:1401-1424.

22. Kreuter A, Krieg T, Worm M, Wenzel J, Moinzadeh P, Kuhn A, et al German guidelines for the diagnosis and therapy of localized scleroderma. J German Soc Dermatol. 2016;14:199-216.

23. Chiu YE. Practice gaps: evaluating the clinical utility of autoantibodies in morphea. JAMA Dermatol. 2013:149:1166.

24. Walker D, Susa JS, Currimbhoy S, Jacobe H. Histopathological changes in morphea and their clinical correlates: results from the morphea in adults and children cohort V. J Am Acad Dermatol. 201/;76:1124-1130.

25. Yamazaki-Nakashimada MA, Saez-De Ocariz M, Maldonado-Colín G García-Romero MT. Subcutaneous immunoglobulin for the treatment of deep morphoea in a child. Clin Exp Dermatol. 2017;43:303-305.

26. Zwischenberger $B$, Jacobe $H$. A systematic review of morphea treatments and therapeutic algorithm. J Am Acad Dermatol. 2011;65:925-941.

27. García-Romero MT, Laxer RM, Pope E. Correlation of clinical tools to determine activity of localized scleroderma in paediatric patients. $\mathrm{Br}$ Dermatol. 2015:174:408-410.

28. Arkachaisri T, Vilaiyuk S, Li S, O’Neil KM, Pope E, Higgins GC, et al. The localized scleroderma skin severity index and physician global assessment of disease activity: a work in progress toward development of localized scleroderma outcome measures. J Rheumatol. 2009;36:2819-2829.

29. Arkachaisri T, Vilaiyuk S, Torok KS, Medsger TA. Development and initial validation of the localized scleroderma skin damage index and physician global assessment of disease damage: a proof-of-concept study. Rheumatology (Oxford). 2010;49:373-381.

30. García-Romero MT, Randhawa HK, Laxer RM, Pope E. The role of loca temperature and other clinical characteristics of localized scleroderma as markers of disease activity. Int J Dermatol. 2016;56:63-67.

31. Khan MA, Shaw L, Eleftheriou D, Prabhakar P, Chong WK, Glover M, et al. Radiologic improvement after early medical intervention in localised facial morphea. Pediatr Dermatol. 2016:33:e95-e98.

32. Brady SM, Shapiro L, Mousa SA. Current and future direction in the management of scleroderma. Arch Dermatol Res. 2016;308:461-471.
33. Hawley DP, Pain CE, Baildam EM, Murphy R, Taylor AE, Foster HE. United Kingdom survey of current management of juvenile localized scleroderma. Rheumatology (Oxford). 2014;53:1849-1854.

34. Warburton KL, McPhee MJ, Savage LJ, Honan AE, Montgomery R, Ghazavi M, et al. Management of morphoea: results of a national survey of U.K. clinicians. Br J Dermatol. 2014;171:1243-1245

35. Fett NM, Werth VP. Update on morphea: part II. Outcome measures and treatment. J Am Acad Dermatol. 2011;64:231-242.

36. Strickland N, Patel G, Strickland A, Jacobe H. Attitudes and trends in the treatment of morphea: a national survey. J Am Acad Dermatol. 2015; 72:727-728.

37. Kreuter A, Altmeyer P, Gambichler T. Treatment of localized scleroderma depends on the clinical subtype. Br J Dermatol. 2007;156:1363-1364.

38. Piram M, McCuaig CC, Saint-Cyr C, Marcoux D, Hatami A, Haddad E, et al. Short- and long-term outcome of linear morphoea in children. $\mathrm{Br} J$ Dermatol. 2013;169:1265-1271.

39. Kroft EB, Groeneveld TJ, Seyger MM, De Jong EM. Efficacy of topical tacrolimus $0.1 \%$ in active plaque morphea: randomized, double-blind controlled pilot study. Am J Clin Dermatol. 2009:10:181-187.

40. Dytoc M, Wat H, Cheung-Lee M, Sawyer D, Ackerman T, Fiorillo L. Evaluation of the efficacy and safety of topical imiquimod $5 \%$ for plaque-type morphea: a multicenter, prospective, vehicle-controlled trial. J Cut Med Surg. 2015;19:132-139.

41. Dytoc MT, Kossintseva I, Ting PT. First case series on the use of calcipotriol-betaethasone dipropionate for morphea. Br J Dermatol. 2007; 157:615-618.

42. Tay YK. Topical calcipotriol ointment in the treatment of morphea. J Dermatolog Treat. 2003;14:219-221.

43. Rodríguez-Castellanos M, Tlacuilo-Parra A, Sánchez-Enriquez S, Vélez-Gómez E, Guevara-Gutiérrez E, et al. Pirfenidone gel in patients with localized scleroderma: a phase II study. Arthr Res Ther. 2015;16:510.

44. Hunzelmann N, Anders S, Fierlbeck G, Hein R, Herrmann K, Albrecht M. Double-blind, placebo-controlled study of intralesional interferongamma fot the treatment of localized scleroderma. J Am Acad Dermatol. 1997;36:433-435.

45. Li S, Torok KS, Pope E, Dedeoglu F, Hong S, Jacobe HT, et al. Development of consensus treatment plans for juvenile localized scleroderma: a roadmap toward comparative effectiveness studies in juvenile localized scleroderma. Arth Care Res (Hoboken). 2012;64:1175-1185.

46. Tratenberg M, Gutwein F, Rao V, Sperber K, Wasserrman A, Ash J. Localized scleroderma: a clinical review. Curr Rheumatol Rev. 2017; 13:86-92.

47. Zulian F, Martini G, Vallongo C, Vittadello F, Falcini F, Patrizi A, et al. Methotrexate treatment in juvenile localized scleroderma: a randomized, double-blind, placebo-controlled trial. Arthritis Rheum. 2011; 63:1998-2006.

48. Zulian F, Vallongo C, Patrizi A, Belloni-Fortina A, Cutrone M, Alessio M, et al. A long-term follow-up study of methotrexate in juvenile localized scleroderma (morphea). J Am Acad Dermatol. 2012:67:1151-1156.

49. Rattanakaemakorn $P$, Jorizzo J. The efficacy of methotrexate in the treatment of en coup de sabre (linear morphea subtype). J Dermatol Treat. 2017;29:197-199.

50. Uziel Y, Feldman B, Krafchik B, Yeung RS, Laxer RM. Methotrexate and corticosteroids therapy for pediatric localized scleroderma. J Pediatr. 2000;136:91-95

51. Weibel L, Sampaio MC, Visentin MT, Howell KJ, Woo P, Harper Jl. Evaluation of methotrexate and corticosteroids for the treatment of localized scleroderma (morphoea) in children. Br J Dermatol. 2006;155:1013-1020.

52. Breuckmann F, Gambichler T, Altmeyer P, Kreuter A. UVA/UVA1 phototherapy and PUVA photochemotherapy in connective tissue diseases and related disorders: a research based review. BMC Dermatol. 2004;4:11.

53. De Rie MA, Enomoto DN, De Vries H, Bos JD. Evaluation of medium-dose UVA-1 phototherapy in localized scleroderma with the cutometer and fast Fourier transform method. Dermatology. 2003;207:298-301.

54. El-Mofty M, Mostafa W, El-Darouty M, Bosseila M, Nada H, Yousef R, et al. Different low doses of broad-band UVA in the treatment of morphea and systemic sclerosis. Photodermatol Photoimmunol Photomed. 2004; 20:148-156.

55. El-Mofty M, Zaher H, Bosseila M, Yousef R, Saad B. Low-dose broadband UVA in morphea using a new method for evaluation. Photodermatol Photoimmunol Photomed. 2000;16:43-49.

56. Keyal U, Bhatta AK, Wang XL. UVA1 a promising approach for scleroderma. Am J Transl Res. 2017:9:4280-4287.

57. Kreuter A, Hyun J, Stücker M, Sommer A, Altmeyer P, Gambichler T. A randomized controlled study of low-dose UVA-1, medium-dose UVA-1 and narrowband UVB phototherapy in the treatment of localized slceroderma. J Am Acad Dermatol. 2006:54:440-447.

58. Pavlotsky F, Sakka N, Lozinski A, Barzilai A. Bath psoralen-UVA photochemotherapy for localized scleroderma: experience from a single institute. Photodermatol Photoimmunol Photomed. 2013;29:247-252.

59. Vásquez R, Jabbar A, Khan F, Buethe D, Ahn C, Jacobe H. Recurrence of morphea after successful ultraviolet A1 phototherapy: a cohort study. J Am Acad Dermatol. 2014;70:481-488 
60. Elst EF, Van Suijlekom-Smit L, Oranje AP. Treatment of linear scleroderma with oral 1,25-dihydroxyvitamin D3 (calcitriol) in seven children. Pediatric Dermatol. 1999;16:53-58.

61. Hulshof MM, Bouwes-Bavinck JN, Bergman W, Masclee AA, Heickendorff $L$, Breedveld FC, et al. Double-blind, placebo controlled study of oral calcitriol for the treatment of localized and systemic scleroderma. J Am Acad Dermatol. 2000;43:1017-1023.

62. Barin EZ, Cinal H, Cakmak MA, Tan O. Treatment of linear scleroderma (en coup de sabre) with dermal fat grafting. J Cutan Med Surg. 2016; 20:269-271.

63. Pimenta de Araujo-Franco J, Soares-Serra M, Barbosa-Lima R, Macedo-D'Acri A, Martins CJ. Scleroderma en coup de sabre treated with polymethylmethacrylate. Case report. An Bras Dermatol. 2016;91:209-211.

64. Ibler KS, Gramkow C, Siemssen PA. Autologous fat transplantation for the treatment of linear scleroderma en coup de sabre. Skinmed. 2015:13:74-76

65. Rimoin L, Arbiser J. Improvement of en coup de sabre morphea and associated headaches with botulinum toxin injections. Dermatol Surg. 2016;42:1216-1219.

66. Íñigo $\mathrm{F}$, Jiménez-Murat $\mathrm{Y}$, Arroyo $\mathrm{O}$, Fernández $\mathrm{M}$, Ysunza A. Restoration of facial contour in Romberg's disease and hemifacial microssomia: experience with 118 cases. Microsurgery. 2000;20:167-172.

67. Palmero ML, Uziel Y, Laxer RM, Forrest CR, Pope E. En coup de sabre scleroderma and Parry-Romberg syndrome in adolescents: surgical options and patient-related outcomes. J Rheumatol. 2010;37:2174-2179.

68. Schoch JJ, Schoch BS, Werthel JD, McIntosh AL, Davis DMR. Orthopedic complications of linear morphea: implications for early interdisciplinary care. Pediatr Dermatol. 2018;35:43-46.

69. Laxer RM, Zulian F. Localized scleroderma. Curr Opin Rheumatol. 2006; 18:606-613.

70. Grundmann-Kollmann M, Ochsendorf F, Zollner TM, Spieth K, Sachsenberg-Studer E, Kaufmann R, et al. PUVA-cream photochemotherapy for the treatment of localized scleroderma. J Am Acad Dermatol. 2000; 43:675-678

71. Shalaby SM, Bosseila M, Fawzy MM, Abdel Halim DM, Sayed SS Allam RS, et al. Fractional carbon dioxide laser versus low-dose UVA-1 phototherapy for treatment of localized scleroderma: a clinical and immunohistochemical randomized controlled study. Laser Med Sci. 2016;31:1707-1715.

72. Usmani N, Murphy A, Veale D, Goulden V, Goodfield M, et al. Photochemotherapy for localized morphea: effect on clinical and molecular markers. Clin Exp Dermatol. 2008;33:698-704.
73. Adeeb F, Anjum S, Hodnett P, et al. Early- and late-stage morphea subtypes with deep tissue involvement is treatable with abatacept (Orencia). Semin Arthr Rheumatism. 2017;46:775-781.

74. Bali G, Fruhauf J, Wutte N, et al. Cyclosporine reduces sclerosis in morphea: a retrospective study in 12 patients and a literature review. Dermatology (Basel, Switzerland). 2016;232:503-510

75. Martini G, Ramanan AV, Falcini F, Girschick H, Goldsmith DP, Zulian F. Successful treatment of severe or methotrexate-resistant juvenile localized scleroderma with mycophenolate mofetil. Rheumatol (Oxford). 2009; 48:1410-1413.

76. Mertens JS, Marsman D, Van de Kerkhof PC, Hoppenreijs EP, Knaapen HK, Radstake TR, et al. Use of mycophenolate mofetil in patients with severe localized scleroderma resistant or intolerant to methotrexate. Act Derm Venereol. 2016;96:510-513.

77. Martini G, Campus S, Raffeiner B, Boscarol G, Meneghel A, Zulian F. Tocilizumab in two children with pansclerotic morphoea: a hopeful therapy for refractory cases? Clin Exp Rheumatol. 2017;106:211-213.

78. Kaur S, Dhar S, Kanwar AJ. Treatment of childhood linear morphea with D-penicillamine. Pediatr Dermatol. 1993:10:201-202.

79. Alcántara-Reifs CM, Garnacho-Saucedo GM, Salido-Vallejo R, De la Corte-Sánchez S, García-Nieto AV. Imatinib treatment of therapy resistant generalized deep morphea. Dermatol Ther. 2015;28:271-273.

80. Coelho-Macías V, Mendes-Bastos P, Assis-Pacheco F, Cardoso J. Imatinib: a novel treatment approach for generalized morphea. Int J Dermatol. 2014;53:1299-1302.

81. Hanson AH, Fivenson DP, Schapiro B. Linear scleroderma in an adolescent woman treated with methotrexate and excimer laser. Dermatol Ther. 2014;27:203-205.

82. Ferguson ID, Weiser $P$, Torok KS. A case report of successful treatment of recalcitrant childhood localized scleroderma with infliximab and leflunomide. Open Rheumatol J. 2015;9:30-35.

83. Shima T, Yamamoto $Y$, Ikeda T, Furukawa F. A patient with localized scleroderma successfully treated with etretinate. Case Rep Dermatol. 2014;6:200-206

84. Pileri A, Raone B, Raboni R, Giudice V, Patrizi A. Generalized morphea successfully treated with extracorporeal photochemotherapy (ECP). Dermatol Online J. 2014;20:21258.

85. Howick J, Chalmers I, Glasziou. Explanation of the 2011 Oxford Centre for Evidence-Based Medicine (OCEBM) levels of evidence. EE. UU.: Oxford Centre for Evidence-Based Medicine; 2011.

86. OCEBM Levels of Evidence Workin Group. The Oxford Levels of Evidence 2. EE. UU.: Oxford Centre for Evidence-Based Medicine; 2009. 


\section{Anexo 1. Niveles de evidencia y grado de recomendación según el Centro de Medicina Basada en Evidencia de Oxford. ${ }^{85,86}$}

\section{Nivel de evidencia}

$1 \mathrm{a}$

$1 \mathrm{~b}$

$1 \mathrm{c}$

$2 a$

$2 \mathrm{~b}$

$2 c$

3a

$3 b$

4

5

\section{Tipo de estudio}

Revisiones sistemáticas (con homogeneidad) de ensayos controlados aleatorizados.

Ensayos controlados aleatorizados individuales (con intervalo de confianza estrecho).

Ensayos controlados aleatorizados tipo todo o nada.

Revisiones sistemáticas (con homogeneidad) de estudios de cohorte.

Estudio de cohorte individual o ensayos controlados aleatorizados de baja calidad (exclusión $>80 \%$ de la muestra).

Búsqueda de resultados; estudios ecológicos.

Revisión sistemática (con homogeneidad) de estudios de casos y controles.

Estudio de casos y controles individual.

Serie de casos (y estudios de casos y controles o de cohorte de baja calidad).

Opinión de expertos sin evaluación crítica explícita, experiencia clínica o basada en fisiología, investigación de banco o "primeros principios".

\section{Grado de eecomendación Significado}

A

Estudios consistentes de nivel 1 (más de uno).

Estudios consistentes de nivel 2 o 3, o extrapolaciones de estudios de nivel 1.

C

Estudios de nivel 4 o extrapolaciones de estudios de nivel 2 o 3.

D 